The multifocal nature of advanced neuroblastoma in humans was fully mimicked in this model

\title{
Escaping those primitive origins
}

Aggressive forms of neuroblastoma often present at diagnosis having already disseminated. The primitive embryonic origin of neuroblastoma from sympathoadrenal neural crest cells has hampered research into the timing and aetiology of the metastatic process in patients and animal models. To overcome these limitations, a group have developed an embryonic avian model that recapitulates the early tumorigenic events and metastasis of neuroblastoma in tissues homologous to those in patients.

The molecular mechanisms that give rise to the multiple foci seen in patients with neuroblastoma remain largely unknown. To address this, Delloye-Bourgeois et al. made an in vivo model by grafting fluorescent stage 4 neuroblastoma cell lines into the sympathoadrenal neural crest of chick embryos. Imaging the embryos in $3 \mathrm{D}$ over time revealed that most of the neuroblastoma cells had migrated away from the graft site by following the same migratory paths and reaching the same target sites (sympathetic ganglia and the adrenal gland) as endogenous sympathoadrenal neural crest cells during development. While migrating, the neuroblastoma cells mirrored the collective migratory patterns characteristic of neural crest cells, forming small migrating masses or streams. Interestingly, neuroblastoma cells were actively proliferating despite exhibiting a migratory phenotype.

Observations over longer time periods (7 days) showed that neuroblastoma cells eventually disseminated away from the primary tumour masses formed in sympathoadrenal regions and invaded as clusters into proximal tissues and distant sites, such as the intes-
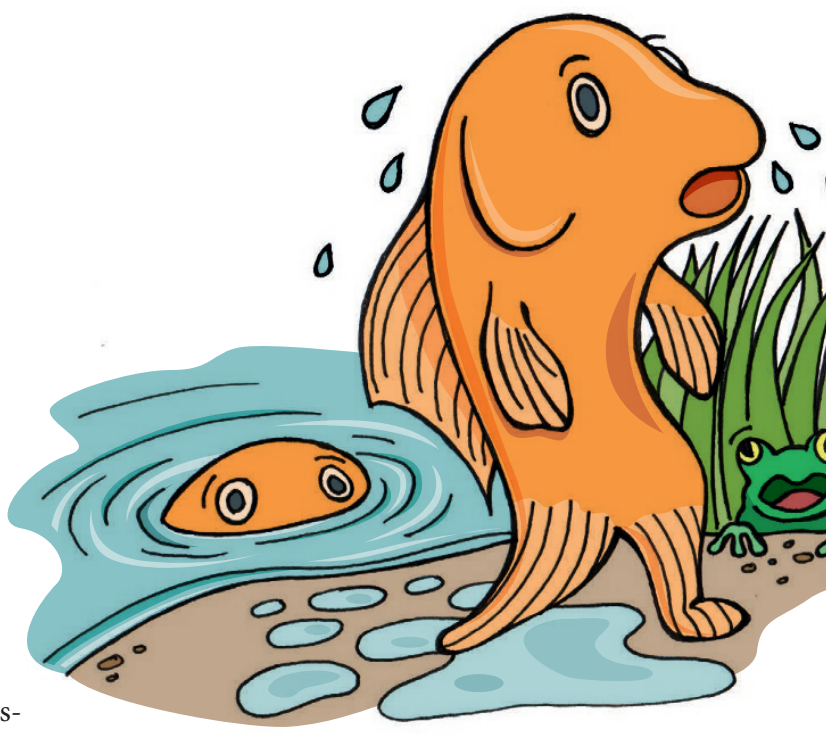

tines and colon.

The multifocal nature of advanced neuroblastoma in humans was fully mimicked in this model and demonstrated that secondary metastasis was achieved either by disseminating via the aorta or along nerves.

The authors next characterized the gene expression profile associated with the migratory behaviour of neuroblastoma cells by comparing the transcriptome of naive neuroblastoma cells, which had not been exposed to the embryonic environment, with that of neuroblastoma cells from primary tumour masses in the sympathetic ganglia. Of the 46 neural crest gene transcripts identified as being differentially expressed, 24 had functions related to cell movement. This included semaphorin 3C (SEMA3C), which was decreased by approximately twofold and chosen for further study, using an inducible system to target SEMA3C with short hairpin RNA (shRNA) in neuroblastoma cell lines. Only if SEMA3C was downregulated after primary tumour formation was the ability of neuroblastoma cells to undergo distant metastasis increased. Grafting a mixed population of high SEMA3C-expressing cells and low SEMA3C-expressing cells into embryos revealed a dose-dependent mechanism of dissemination. Specifically, SEMA3C functions as a paracrine cohesive signal, necessary for the initial migration towards sympathoadrenal targets, wherein its downregulation serves as an autocrine signal to mediate distant metastasis. Additional work demonstrated that the propensity for cohesion between neuroblastoma cells mediated by SEMA3C is also transcriptionally regulated by components of canonical receptor complexes for secreted semaphorins, neuropilin 1 (NRP1), NRP2 and plexin A4 (PLXNA4).

As low SEMA3C expression correlated with metastatic disease and poor survival in patients with neuroblastoma, Delloye-Bourgeois et al. developed their avian model further by grafting tumour biopsies from localized tumour resections and bone marrow aspirates from patients with metastases into chick embryos. These patient-derived xenografts showed tumour formation at sympathoadrenal sites, independent of the sample origin, and metastatic spread only in cases where tumours were derived from metastases.

Given the poor performance of existing mouse models of neuroblastoma, this study highlights the strength of an unconventional avian model to shed light on the early events triggering metastatic dissemination.

Anna Dart

ORIGINAL ARTICLE Delloye-Bourgeois, C. et al. Microenvironment-driven shift of cohesion/ detachment balance within tumors induces switch toward metastasis in neuroblastoma. Cancer Cell 32, 427-443 (2017) 